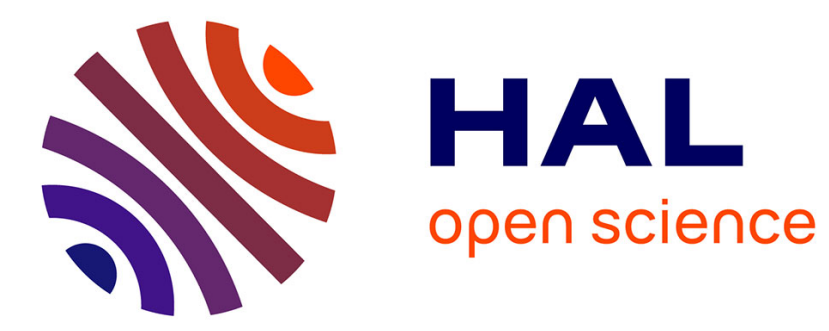

\title{
Towards interval-based non-additive deconvolution in signal processing
}

\author{
Olivier Strauss, Agnès Rico
}

\section{To cite this version:}

Olivier Strauss, Agnès Rico. Towards interval-based non-additive deconvolution in signal processing. Soft Computing, 2012, 16 (5), pp.809-820. 10.1007/s00500-011-0771-7 . lirmm-00857417

\section{HAL Id: lirmm-00857417 https://hal-lirmm.ccsd.cnrs.fr/lirmm-00857417}

Submitted on 10 Sep 2013

HAL is a multi-disciplinary open access archive for the deposit and dissemination of scientific research documents, whether they are published or not. The documents may come from teaching and research institutions in France or abroad, or from public or private research centers.
L'archive ouverte pluridisciplinaire HAL, est destinée au dépôt et à la diffusion de documents scientifiques de niveau recherche, publiés ou non, émanant des établissements d'enseignement et de recherche français ou étrangers, des laboratoires publics ou privés. 


\title{
Towards interval-based non-additive deconvolution in signal processing
}

\author{
Olivier Strauss and Agnès Rico \\ LIRMM Université Montpellier II, 61 rue Ada, 34392 Montpellier cedex 5, France \\ ERIC Université Claude Bernard Lyon 1, 43 bld du 11 novembre 1918, 69622 \\ Villeurbanne, France \\ strauss@lirmm.fr, agnes.rico@univ-lyon1.fr
}

\begin{abstract}
Reconstructing a signal from its observations via a sensor device is usually called "deconvolution". Such reconstruction requires perfect knowledge of the impulse response of the sensor involved in the signal measurement. The lower this knowledge, the more biased the reconstruction. In this paper, we present a novel method for reconstructing a signal measured by a sensor whose impulse response is imprecisely known. This technique is based on modeling the relationship between the measurement and the signal via a concave capacity and extending the convolution concept to a concave set of impulse responses. The reconstructed signal is interval-valued, thus reflecting the poor knowledge of the sensor impulse response.
\end{abstract}

Keywords: inverse problem, deconvolution, non-additive confidence measure, Choquet integral, Schultz iterative procedure

\section{Introduction}

Deconvolution consists of reconstructing a signal from its noisy measurements. It has numerous applications in many scientific and engineering disciplines: recovering earth structures in seismology [6], reversing optical distortion in microscopy, deblurring satellite images [11], tomography [7,19], etc. Generally, deconvolution involves finding a solution to a convolution equation of the form:

$$
m(t)=(h \otimes s)(t)+b(t),
$$

where $s(t)$ is the signal, $m(t)$ its measurement, $h(t)$ the sensor impulse response and $b(t)$ the measurement error due to transmission, thermal noise, sampling, quantification, etc. Note that $b(t)$ is often referred to as a random variable having a known distribution (usually Gaussian). Roughly speaking, the inversion of equation (1) is an attempt to find a linear deconvolution operator $\otimes^{-1}$ of an inverse $h^{-1}$ of the impulse response:

$$
\hat{s}(t)=\left(h \otimes^{-1} m\right)(t)=\left(h^{-1} \otimes m\right)(t) .
$$

The proposed deconvolution operator usually does not exist as a bounded linear operator. Therefore, the solution to (1) is not unique, may not exist and its 
computation can lead to very unstable solutions. Moreover, the deconvolution process tends to interpret noise $b(t)$ in a coherent manner, leading to artifacts added to the reconstructed signal:

$$
\hat{s}(t)=\left(h \otimes^{-1} h \otimes s\right)(t)-\left(h \otimes^{-1} b\right)(t),
$$

where $\left(h \otimes^{-1} b\right)(t)$ represents these additive artifacts. Even if $b(t)$ is nice white noise, the artifacts are not. This study will be restricted to positive impulse responses. It should be further extended to impulse responses having both positive and negative values.

Instead of trying and find a continuous solution to Equation (1), deconvolution is usually performed in the discrete domain (even if the convolution relation is defined in the continuous domain). Discrete deconvolution consists of solving a matrix equation of the form:

$$
M=A S+B
$$

where $M$ is the vector of sampled measures, $S$ is the vector of the sampled signal, $B$ is a noise vector and $A$ is a matrix depending on the impulse response of the acquisition system. Deconvolution thus consists of solving the matrix equation (4) by minimizing a risk function [13]. A very common risk function is the Euclidian distance: $J_{A}(S, M)=\|M-A S\|^{2}$. Minimizing this risk function leads to computing the following solution:

$$
\hat{S}=A^{+} M,
$$

where $A^{+}$is the pseudo inverse matrix of $A$.

When the measurements are noisy, then performing the deconvolution by using Equation (5) induces reconstruction artifacts. A solution to minimize these artifacts generally involves modifying the reconstruction criterion by adding a regularizing term in order to limit the reconstructed signal dynamics. Because of the complexity of the regularization or of the high dimension of $A$, most reconstruction algorithms are iterative, i.e. starting from a wrong solution, converge to a solution that minimizes the proposed criterion. Most approaches proposed in the relevant literature focus on noise in the measurement and assume that the blur operator ( $h$ or $A$ ) is known [16]. However, since knowledge on $A$ generally comes from an identification process, this hypothesis is usually violated. Moreover, Equation (4) is usually an approximation of the true relation. A lack of knowledge on the blur operator is generally considered as being a measurement error [17], leading to biased reconstructed signals with a systematic error that is not quantified. A conventional method for dealing with this problem is to consider matrix $A$ as being interval-valued [12]. This approach leads to guaranteed but not very specific inversion of the interval extension of Equation (4). Guaranteed means that, if the interval-valued matrix contains the real matrix, then the signal is included in the interval-valued reconstructed signal. However, this method is very computationally expensive and the guarantee relies on the ability to predict an appropriate bound of the reconstruction error. In a previous paper [18], we proposed an alternative method that uses a capacity $\nu$ to 
model imprecise knowledge of the impulse response of a sensor. This modeling entails a generalized convolution operator based on the Choquet integral leading to modeling the measurement process by a non-linear equation of the form:

$$
[\underline{M}, \bar{M}]=\sigma \cdot \mathcal{A}_{\nu}(S)
$$

where $\underline{M}$ (rsp. $\bar{M})$ is the lower bound (rsp. upper bound) of any measurement vector that should have been obtained by using one of the impulse responses modeled by capacity $\nu$ and a real coefficient $\sigma$. Modeling the imprecise knowledge with a concave capacity has two main advantages. First, it leads to a very specific estimate of the convex envelope of all output signals that should have been obtained by convoluting the input signal with all impulse responses represented by this modeling. Second, it leads to an algorithmic implantation whose computational complexity is as low as that of a traditional linear convolution operation.

This paper aims at proposing an inversion scheme of Equation (6). More precisely, given $M$ the actual measurement vector, we aim at finding a specific convex set $[\underline{S}, \bar{S}]$ in agreement with $M$ via Equation (6), that is find $[\underline{S}, \bar{S}]$ such that:

$$
\forall S \in[\underline{S}, \bar{S}], M \in \sigma \cdot \mathcal{A}_{\nu}(S) .
$$

This article is organized as follows: section 2 presents the framework and notations. This section is divided into six subsections to explain how a lack of knowledge on the impulse response can be modeled by a capacity. Section 3 presents the interval-valued deconvolution as an extension of the Schultz iterative procedure. Section 4 presents some illustrative experiments. Section 5 is devoted to our concluding remarks.

\section{Framework and notations}

\subsection{Filtering seen as an expectation operator}

Let $S=\left(s_{i}\right)_{i \in\{1, \ldots, N\}} \in \mathbb{R}$ be a real discrete signal composed of $N$ samples. $\{1, \ldots, N\}$ will be denoted $\Omega$. Filtering $S$ consists of convoluting the set of all sampled values with a particular discrete function called the impulse response of the filter $h=\left(h_{i}\right)_{i \in \mathbb{Z}}$. In this paper, we only consider positive impulse responses. The digital filter output is a sequence of $N$ sampled values denoted $M=\left(m_{k}\right)_{k \in\{1, \ldots, N\}}$. The computation of $m_{k}$, the $k^{t h}$ output of the filter, is given by:

$$
m_{k}=\sum_{i=1}^{N} s_{i} h_{k-i} .
$$

Let $\sigma=\sum_{i \in \mathbb{Z}} h_{i}, \rho_{i}=\frac{h_{i}}{\sigma}$, and $\rho=\left(\rho_{i}\right)_{i \in \mathbb{Z}} \cdot \rho$ can be considered as a discrete probability distribution defining a probability measure $P$. Let $\rho^{k}=\left(\rho_{i}^{k}\right)_{i \in \mathbb{Z}}$ be 
the probability distribution defined by translating $\rho: \rho_{i}^{k}=\rho_{k-i}$, hence Equation (8) can be re-written as follows:

$$
m_{k}=\sigma \sum_{i=1}^{N} s_{i} \rho_{i}^{k}=\sigma \mathbb{E}_{P_{k}}(S),
$$

where $P_{k}$ is the probability measure defined by the translated probability distribution $\rho^{k}$ and $\mathbb{E}_{P_{k}}$ is the expectation operator induced by $P_{k}$ on sampled values of $\Omega$. Thus, filtering a signal with a linear filter whose impulse response is positive can be seen as an expectation operation multiplied by a constant real value. This operation can be presented in matrix form:

$$
M=\sigma A_{P} S,
$$

where $M$ and $S$ respectively denote the measurement and the input signal vector, and with $A_{P}$ defined by:

$$
A_{P}=\left[\begin{array}{cccc}
\rho_{0}^{0} & \rho_{1}^{0} & \ldots & \rho_{N}^{0} \\
\rho_{0}^{1} & \rho_{1}^{1} & \ldots & \rho_{N}^{1} \\
\ldots & \ldots & \ldots & \ldots \\
\rho_{0}^{N} & \rho_{1}^{N} & \ldots & \rho_{N}^{N}
\end{array}\right]=\left[\begin{array}{cccc}
\rho_{0} & \rho_{-1} & \ldots & \rho_{-N} \\
\rho_{1} & \rho_{0} & \ldots & \rho_{-N+1} \\
\ldots & \ldots & \ldots & \ldots \\
\rho_{N} & \rho_{N-1} & \ldots & \rho_{0}
\end{array}\right]
$$

\subsection{An interval-valued generalization of the expectation operator}

This section proposes an interval-valued generalization of the conventional expectation operator. It sums up different results presented in [18]. It is based on replacing the usual probability measure by a more general confidence measure called a capacity (see e.g. [1]). The use of a capacity to represent a confidence measure entails using a more general expectation operator called the Choquet integral (see [2]).

This part begins with some necessary definitions and properties concerning the capacities and the Choquet integral.

Let $\Omega=\{1, \ldots, N\}$ be a finite subset of $\mathbb{N}$ (which can be considered here as a set of indices corresponding to the signal samples), while $\mathcal{P}(\Omega)$ is the set of all subsets of $\Omega$ and $V$ is the set all the real functions defined on $\Omega$. Note that $X \in V$ can also be considered as a vector $X=\left(x_{1}, \ldots, x_{N}\right)^{T}$, where $(.)^{T}$ is the transposition operator.

Definition $1 A$ capacity $\nu$ is a set function $\nu: \mathcal{P}(\Omega) \rightarrow[0,1]$ such that $\nu(\varnothing)=$ $0, \nu(\Omega)=1$, and $\forall A \subseteq B \Rightarrow \nu(A) \leq \nu(B)$.

Given a capacity $\nu$, its conjuguate $\nu^{c}$ is defined as: $\nu^{c}(A)=1-\nu\left(A^{c}\right)$, for any subset $A$ of $\Omega$, with $A^{c}$ being the complementary set of $A$ in $\Omega$. A capacity $\nu$ such that $\forall A, B \in \mathcal{P}(\Omega), \nu(A \cup B)+\nu(A \cap B) \leq \nu(A)+\nu(B)$ is

\footnotetext{
${ }^{1}$ The conventional $\bar{\nu}$ notation will not be used in this paper so as to make the equations below more easily understandable.
} 
said to be concave. If a capacity is concave, its conjugate is convex, i.e. $\forall A, B \in$ $\mathcal{P}(\Omega), \nu^{c}(A \cup B)+\nu^{c}(A \cap B) \geq \nu^{c}(A)+\nu^{c}(B)$

The core of a capacity ${ }^{2} \nu$, denoted $\operatorname{core}(\nu)$, is the set of probability measures $P$ defined on $\Omega$ such that $\forall A \in \Omega, \nu(A) \geq P(A)$.

Definition 2 Let $\nu$ be a capacity on $\mathcal{P}(\Omega)$, and $X \in V$ be a finite positive real function, then the Choquet integral of $X$ with respect to $\nu$ is defined by:

$$
C_{\nu}(X)=\sum_{n=1}^{N} x_{(n)}\left(\nu\left(A_{(n)}\right)-\nu\left(A_{(n+1)}\right)\right),
$$

where (.) indicates a permutation that sorts the $x_{n}$ in increasing order: $x_{(1)} \leq$ $\ldots \leq x_{(N)}$, with subsets $A_{(i)}$ being defined by: $A_{(i)}=\{(i), \ldots,(N)\}$, and $A_{(N+1)}=$ $\varnothing$.

The standard Choquet integral is defined for positive functions. Since our approach is dedicated to filtering signals that can take negative values, we will use the standard extension of the Choquet integral [9].

Let $X \in V$ be a real function. Let us define the two real functions $X^{+} \in V$ and $X^{-} \in V$ by: $X^{+}=\max (X, 0)$ and $X^{-}=\max (-X, 0)$, where 0 denotes the function equal to 0 everywhere and $\max (X, Y)$ denotes the point-wise maximum of $X$ and $Y$. By construction, $X^{-}$and $X^{+}$are positive real functions and $X=$ $X^{+}-X^{-}$.

Definition 3 Let $X \in V$ be a real function and $\nu$ be a capacity on $\mathcal{P}(\Omega)$, then the asymmetric Choquet integral of $X$ with respect to $\nu$ is defined by:

$$
\check{C}_{\nu}(X)=C_{\nu}\left(X^{+}\right)-C_{\nu^{c}}\left(X^{-}\right)
$$

An important result, proved by [3], will be used hereafter:

Theorem 1 If $\nu$ is a concave capacity on $\mathcal{P}(\Omega)$, then for all $X \in V, \check{C}_{\nu^{c}}(X)=$ $\inf _{P \in \operatorname{core}(\nu)} \mathbb{E}_{P}(X)$ and $\check{C}_{\nu}(X)=\sup _{P \in \operatorname{core}(\nu)} \mathbb{E}_{P}(X)$, where $\mathbb{E}_{P}$ is the standard expectation based on the probability measure $P$.

Thus, if $\nu$ is a concave capacity, then $\forall X \in V$ and $\forall P \in \operatorname{core}(\nu)$ we have $\check{C}_{v^{c}}(X) \leq \mathbb{E}_{P}(X) \leq \check{C}_{v}(X)$. The interval-valued extension of the expectation operator we propose is based on this property.

Definition 4 Let $\nu$ be a concave capacity defined on $\mathcal{P}(\Omega)$ and $X \in V$ be a real function on $\Omega$, then the imprecise expectation of $X$ with respect to $\nu$ is defined by: $\overline{\mathbb{E}}_{\nu}(X)=\left[\check{C}_{\nu^{c}}(X), \check{C}_{\nu}(X)\right]$

\footnotetext{
${ }^{2}$ In [20] the core is defined for a convex capacity. Our definition coincides with the definition proposed in [3] considering its conjugate (concave) capacity.
} 
Based on Theorem 1, this extended expectation operator satisfies the property: $\forall P \in \operatorname{core}(\nu), \mathbb{E}_{P}(X) \in \underline{\mathbb{E}}_{\nu}(X)$. It is an extension since a probability measure is simply an additive capacity (i.e. a capacity that is concave and convex) and the imprecise valued expectation coincides with the usual precise valued expectation when the considered capacity is a probability measure: $\underline{\mathbb{E}}_{P}=\mathbb{E}_{P}$. See [18] for more details.

We will also need to consider an extension of this interval-valued operator to interval-valued functions. Let $I V$ be the set of all interval-valued functions on $\Omega$. $[\mathbf{X}] \in I V$ can be seen as an interval-valued vector defined by: $[\mathbf{X}]=$ $\left(\left[\underline{x_{1}}, \overline{x_{1}}\right], \cdots,\left[\underline{x_{N}}, \overline{x_{N}}\right]\right)^{T}$ where $\forall i,\left[\underline{x_{i}}, \overline{x_{i}}\right]$ is a real interval denoted $\left[\mathbf{x}_{i}\right]$.

Such an interval valued vector can also be defined by two real vectors $\underline{X}=$ $\left(\underline{x_{1}}, \ldots, \underline{x_{N}}\right)^{T}$ and $\bar{X}=\left(\overline{x_{1}}, \ldots, \overline{x_{N}}\right)^{T}$. We will thus denote an interval-valued vector $[\overline{\mathbf{X}}]$ by: $[\mathbf{X}]=[\underline{X}, \bar{X}]$.

The standard precise valued expectation operator can be easily extended to interval-valued functions (see [4]), i.e. with $P$ being a probability measure on $\Omega$ and $[\mathbf{X}]=[\underline{X}, \bar{X}] \in I V$ being an interval valued function, then $\mathbb{E}_{P}([\mathbf{X}])$ can be defined by: $\mathbb{E}_{P}([\mathbf{X}])=\left[\mathbb{E}_{P}(\underline{X}), \mathbb{E}_{P}(\bar{X})\right]$. It verifies: $\forall Y \in[\mathbf{X}], \mathbb{E}_{P}(Y) \in \mathbb{E}_{P}([\mathbf{X}])$ and $\forall W \in \mathbb{E}_{P}([\mathbf{X}]), \exists Y \in[\overline{\mathbf{X}}]$ such that $W=\mathbb{E}_{P}(Y)$.

The asymmetric Choquet integral is an increasing function, thus if $\nu$ is a concave capacity: $\underline{X} \leq \bar{X}$ entails $\check{C}_{\nu^{c}}(\underline{X}) \leq \check{C}_{\nu}(\bar{X})$. The imprecise valued expectation we propose can thus be easily extended to an interval-valued function by considering the union of all precise valued expectations: $\forall[\mathbf{X}] \in I V$, $\underline{\mathbb{E}}_{\nu}([\mathbf{X}])=\bigcup_{P \in \operatorname{core}(\nu)} \mathbb{E}_{P}([\mathbf{X}])$. Computation of this interval-valued expectation takes advantage of the fact that the asymmetric Choquet integral is increasing.

Definition 5 Let $\nu$ be a concave capacity on $\mathcal{P}(\Omega)$ and $[\mathbf{X}]=[\underline{X}, \bar{X}] \in I V$ be an interval valued function on $\Omega$, then the imprecise expectation operator of $[\mathbf{X}]$ with respect to $\nu$ can be computed by: $\underline{\mathbb{E}}_{\nu}([\mathbf{X}])=\left[\check{C}_{\nu^{c}}(\underline{X}), \check{C}_{\nu}(\bar{X})\right]$.

Proof: with $\nu$ being concave, for all $P \in \operatorname{core}(\nu)$, we have $\forall[\mathbf{X}] \in I V$, $\mathbb{E}_{P}([\mathbf{X}]) \subseteq\left[\check{C}_{\nu^{c}}(\underline{X}), \check{C}_{\nu}(\bar{X})\right]$ which entails $\bigcup_{P \in \operatorname{core}(\nu)} \mathbb{E}_{P}([\mathbf{X}]) \subseteq\left[\check{C}_{\nu^{c}}(\underline{X}), \check{C}_{\nu}(\bar{X})\right]$.

Now let $m \in\left[\check{C}_{\nu^{c}}(\underline{X}), \check{C}_{\nu}(\bar{X})\right]$, as $\forall X \in V, \check{C}_{\nu^{c}}(X)=\inf _{P \in \operatorname{core}(\nu)} \mathbb{E}_{P}(X)$ and $\check{C}_{\nu}(X)=\sup _{P \in \operatorname{core}(\nu)} \mathbb{E}_{P}(X)$, there are two probability measures $P_{0}$ and $P_{1} \in \operatorname{core}(\nu)$ such that $\mathbb{E}_{P_{0}}(\underline{X}) \leq m \leq \mathbb{E}_{P_{1}}(\bar{X})$. Now there are two cases. Either $\mathbb{E}_{P_{0}}(\underline{X}) \leq m \leq \mathbb{E}_{P_{0}}(\bar{X})$ and then $\left[\check{C}_{\nu^{c}}(\underline{X}), \check{C}_{\nu}(\bar{X})\right] \subseteq \bigcup_{P \in \operatorname{core}(\nu)} \mathbb{E}_{P}([\mathbf{X}])$ and the property is verified. Otherwise $\mathbb{E}_{P_{0}}(\bar{X})<m \leq \mathbb{E}_{P_{1}}(\bar{X})$ and, due to the continuity, $\exists P_{2} \in \operatorname{core}(\nu)$ such that $m=\mathbb{E}_{P_{2}}(\bar{X})$. In this case, $\exists P \in \operatorname{core}(\nu)$ such that $m \in \mathbb{E}_{P}([\mathbf{X}])$ which concludes the proof.

\subsection{Interval-valued vector arithmetic}

The Minskowski addition is the natural generalization of the conventional addition of real vectors to real interval-valued vectors. Let + be the conventional addition between vectors, i.e. $\forall X, Y \in V, Z=X+Y$ means that $\forall n \in \Omega$, 
$z_{n}=x_{n}+y_{n}$. Let $[\mathbf{X}]=[\underline{X}, \bar{X}]$ and $[\mathbf{Y}]=[\underline{Y}, \bar{Y}]$ be two interval valued vectors, then their Minkowski addition $[\mathbf{Z}]=[\mathbf{X}] \oplus[\mathbf{Y}]$ is defined by:

$$
[\mathbf{Z}]=[\mathbf{X}] \oplus[\mathbf{Y}]=[\underline{X}+\underline{Y}, \bar{X}+\bar{Y}] .
$$

A dual extension of the addition of real vectors can be defined under the name dual Minkowski addition by:

$$
[\mathbf{Z}]=[\mathbf{X}] \boxplus[\mathbf{Y}]=[\min (\underline{X}+\bar{Y}, \bar{X}+\underline{Y}), \max (\underline{X}+\bar{Y}, \bar{X}+\underline{Y})],
$$

where the $\min (A, B)$ operator (resp. $\max (A, B)$ ) is the pointwise minimum (resp. maximum) of two vectors $A$ and $B$. This dual addition is defined according to the previous one in the sense that it is the point-wise solution of a set of two equations involving the Minkowski addition, i.e.: $[\mathbf{X}]=[\mathbf{Z}] \oplus[-\mathbf{Y}]$ and $[\mathbf{Y}]=[\mathbf{Z}] \oplus[-\mathbf{X}]$. In fact, when considering the $n^{\text {th }}$ coordinates of these two equations, only one of the obtained equations $\left[\mathbf{x}_{\mathbf{n}}\right]=\left[\mathbf{z}_{\mathbf{n}}\right] \oplus\left[-\mathbf{y}_{\mathbf{n}}\right]$ and $\left[\mathbf{y}_{\mathbf{n}}\right]=\left[\mathbf{z}_{\mathbf{n}}\right] \oplus\left[-\mathbf{x}_{\mathbf{n}}\right]$ has a solution and it is given by $\left[\mathbf{z}_{\mathbf{n}}\right]=\left[\mathbf{x}_{\mathbf{n}}\right] \boxplus\left[\mathbf{y}_{\mathbf{n}}\right]$.

\subsection{The Schultz iterative procedure}

As explained in the introductory part of the paper, the least squares inversion of Equation (4) can be obtained by computing $A^{+}$the pseudo-inverse of the matrix $A: \hat{S}=A^{+} M . \hat{S}$ is the standard solution of the regularized equation $\left(A^{T} A\right) S=A^{T} M$. In fact, if matrix $A^{T} A$ is well conditioned and of reasonable size, then $A^{+}$can be computed by $A^{+}=\left(A^{T} A\right)^{-1} A^{T}$. Recursive procedures like the Gréville algorithm can also be used. Conversely, if $\left(A^{T} A\right)$ is ill-conditioned or if its size is too huge, then the direct estimation of $\hat{S}$ by computing $A^{+}$ has to be replaced by other procedures. One of these methods is the Schultz iterative procedure (often called the Hotelling iterative procedure, see [10]). This method has been extensively used for inverting ill-conditioned problems, e.g. for tomographic reconstruction [8]. Starting from a wrong solution (e.g. $S^{0}=0$ ), the Schultz procedure iteratively corrects this value and converges towards the least squares solution. The computation of the Schultz procedure is given by:

$$
S^{i+1}=S^{i}+R\left(M-A S^{i}\right),
$$

where $S^{i}$ is the estimation of $S$ at the $i^{\text {th }}$ iteration and $R$ is an estimate of the pseudo-inverse matrix $A^{+}$. Such an approximation can be obtained by: $R=$ $\lambda D A^{T}$, where $D$ is the diagonal matrix composed of the inverse elements of the diagonal elements of $\left(A^{T} A\right)$ and $\lambda$ is a positive real. The convergence of $S^{i}$ towards $\hat{S}$ is known to be slow but is guaranteed for $\lambda \in(0,1][5]$.

In this article, we aim at inverting a non-linear interval-valued measurement equation of the form of Equation (6). Our proposition is to extend the Schultz iterative procedure to this interval-valued equation. This extension needs a partial re-interpretation of the Schultz iterative procedure including considering set additive and subtractive operations. 


\subsection{Matrix $A$ and the impulse response $h$ : a continuous to discrete relation}

Usually, when considering that the sampling frequency is very high compared to the highest frequency of the input signal, matrix $A$ involved in Equation (4) can be defined by the sampled values of the continuous impulse response $h$. Let $T$ be the sampling period, then matrix $A$ is defined by:

$$
A=\sigma_{h} \cdot A_{P}
$$

where $\sigma_{h}=\sum_{i=-\infty}^{\infty} h(i T), \forall i, \rho_{i}=\sigma_{h}^{-1} h(i T)$, then $A_{P}$ is the matrix defined by (11) and $P$ is the probability measure associated with $\left(\rho_{i}\right)_{i \in \mathbb{Z}}$.

However, this situation is not very realistic. Even analog-to-digital converters with a high sampling frequency have a non-negligible impulse response, since the measurement consists of integrating the signal over a short period. Thus the relation between the continuous and the discrete model is better modeled by a sampling kernel $\kappa[21]$. Within this new modeling, the values $h(i T)$ in equation (15) have to be replaced by $\left(h \otimes \kappa^{i T}\right)(0)$, with $\kappa^{i T}$ being the kernel $\kappa$ translated in $i T$ and defined by: $\kappa^{i T}(t)=\kappa(i T-t)[14]$ and $\otimes$ being the convolution operator. When the conversion is considered as being transparent, then we consider $h \otimes \kappa$ instead of $h$ in the digital signal processing, which can lead to a consequent bias, particularly in iterated signal processing or inversion.

Finally, matrix $D$ used in the Schultz procedure (Equation (14)) to define an approximation of matrix $A^{+}$will be simply: $D=\frac{1}{\sum_{i=-\infty}^{\infty} h^{2}(i T)} \cdot I d_{N}$, which can be approximated by $D=\sigma_{h}^{-2} \cdot I d_{N}$, with $I d_{N}$ being the $N \times N$ identity matrix. Thus, matrix $R$ is given by $R=\lambda \sigma_{h}^{-2} A^{T}$.

\subsection{Imprecise filtering}

As noted in section 2.1, when the impulse response of the filter is positive, then the filtering procedure can be seen as a linear aggregation. Let us consider Equation (9). Let $\nu$ be the concave capacity dominating $P$, i.e. the probability measure induced by $\rho$, thus the capacity $\nu_{k}$, which is the capacity $\nu$ translated in $k$, dominates $P_{k}$, which is the probability measure induced by $\rho^{k}$. Therefore, by simply substituting $\mathbb{E}_{P_{k}}$ by $\underline{\mathbb{E}}_{\nu_{k}}$ in Equation (9), we thus define an imprecise filtering process by:

$$
\left[\underline{m_{k}}, \overline{m_{k}}\right]=\sigma \overline{\mathbb{E}}_{\nu_{k}}(S) \text {. }
$$

This operator satisfies the following property: $m_{k}=\sigma \mathbb{E}_{P_{k}}(S) \in\left[\underline{m_{k}}, \overline{m_{k}}\right]$.

Let us now suppose that the impulse function is known but that the kernel that ensures the continuous to discrete interplay is unknown. In that case, it is possible to define a capacity whose core is the set of all discrete kernels that would have been obtained by convoluting a sampling kernel with the known continuous impulse response. How this capacity is built is explained in [18].

Let us define $[\mathbf{M}]$ the interval valued vector by : $[\mathbf{M}]=\left(\left[\underline{m_{1}}, \overline{m_{1}}\right], \ldots,\left[\underline{m_{N}}, \overline{m_{N}}\right]\right)$, then all imprecise filtering operations defining $[\mathbf{M}]$ can be denoted by: 


$$
[\mathbf{M}]=\sigma \mathcal{A}_{\nu}(S) .
$$

As the asymmetric Choquet integral is an increasing function, this imprecise filtering operator can be easily extended to interval valued input signals by simply replacing $S$ (the precise valued vector) by $[\mathbf{S}]$ (an interval valued vector): $[\mathbf{M}]=\sigma \mathcal{A}_{\nu}([\mathbf{S}])$, which is a condensed notation for: $\forall k \in \Omega,\left[\underline{m_{k}}, \overline{m_{k}}\right]=$ $\sigma \overline{\mathbb{E}}_{\nu_{k}}([\mathbf{S}])$.

In the same manner, we defined $\operatorname{core}(\nu)$ as a convex set of probability measures, we define $\operatorname{core}\left(\mathcal{A}_{\nu}\right)$ as the set of all linear operators associated with a matrix $A_{P}$ (equation (11)) where $P$, the probability measure induced by $\rho$, belongs to core $(\nu)$. We also define $\nu^{T}$ as being the capacity that dominates $\rho^{T}$, the probability distribution that induces the probability measure associated with the matrix $A_{P}^{T}\left(\rho_{i}^{T}=\rho_{-i}\right)$.

\section{Interval valued deconvolution}

In this section, we propose to try and invert in a certain sense the linear equation (17) by extending the Schultz iterative procedure. The non-linear iterative procedure we obtain is based on an alternative interpretation of Equation (14). This interpretation means that, at the $(i+1)^{t h}$ iteration, the best estimate of $\Delta^{i}$, which is the additive update of $S^{i}$ to obtain an estimate $S^{i+1}$ closer to $\hat{S}$ than $S^{i}$, is given by:

$$
\Delta^{i}=R\left(M-A S^{i}\right)=S^{i+1}-S^{i} .
$$

In other words, $S^{i+1}$ is the solution of Equation (18). By construction, $A=$ $\sigma_{h} A_{P}$ and $R=\lambda \sigma_{h}^{-2} A^{T}$, thus:

$$
\Delta^{i}=\lambda \sigma_{h}^{-2} \cdot A_{P}^{T} \cdot\left(M-\sigma_{h} A_{P} S^{i}\right) .
$$

\subsection{Definition}

Let $M$ be the actual measured vector. In the same way as the Schultz iterative procedure builds a sequence of estimates $S^{i}$ such that $A S^{i+1}$ is closer to $M$ than $A S^{i}$, we aim at building a sequence of interval valued estimates $\left[\mathbf{S}^{i}\right]$ such that $\sigma_{h} \mathcal{A}_{\nu}\left(\left[\mathbf{S}^{i+1}\right]\right)$ is closer to $M$ than $\sigma_{h} \mathcal{A}_{\nu}\left(\left[\mathbf{S}^{i}\right]\right)$. We aim at obtaining a solution $[\mathbf{S}]=[\underline{S}, \bar{S}]=\lim _{i \rightarrow \infty}\left[\mathbf{S}^{i}\right]$ that corresponds to the convex hull of all the least squares solutions of $\lambda \sigma_{h} A_{P}(S)=M$ with $P \in \operatorname{core}(\nu)$.

The principle of the solution we propose is very simple. Let $\left[\mathbf{S}^{i}\right]$ be the estimated interval at the $i^{t h}$ iteration. We can thus compute the interval $\left[\mathbf{M}^{i}\right]=$ $\left[\underline{M}^{i}, \bar{M}^{i}\right]$ which is a specific as possible convex hull of all the values $\sigma_{h} A_{P}(S)$ with $S \in\left[\mathbf{S}^{i}\right]$ and $P \in \operatorname{core}(\nu)$ by: $\left[\mathbf{M}^{i}\right]=\sigma_{h} \cdot \mathcal{A}_{\nu}\left(\left[\mathbf{S}^{i}\right]\right)$.

Let $\left[\boldsymbol{\Delta}^{i}\right]=\lambda \sigma_{h}^{-2} \mathcal{A}_{\nu^{T}}\left(M-\sigma_{h} \mathcal{A}_{\nu}\left(\left[\mathbf{S}^{i}\right]\right)\right)$. By construction, $\left[\boldsymbol{\Delta}^{i}\right]=\{\Delta=$ $\left.\lambda \sigma_{h}^{-2} \cdot A_{Q}^{T} \cdot\left(M-\sigma_{h} A_{P} S\right), S \in\left[\mathbf{S}^{i}\right], P, Q \in \operatorname{core}(\nu)\right\}$. A first approach to generalize the Schultz procedure would consist of setting $\left[\mathbf{S}^{i+1}\right]=\left[\mathbf{S}^{i}\right] \oplus\left[\boldsymbol{\Delta}^{i}\right]$. This 
solution is not appropriate since the use of the Minkowski addition $\oplus$ would lead to a sequence of interval-valued solutions whose length increases with the iterations. The obtained solution would be completely non-specific, and therefore uninformative. Based on the interpretation we gave on the Schultz procedure, we can say that $\left[\mathbf{S}^{i+1}\right]$ is the set of all solutions of $\Delta=S-B$ either for all $B \in\left[\mathbf{S}^{i}\right]$ and at least one $\Delta \in\left[\boldsymbol{\Delta}^{i}\right]$ or for all $\Delta \in\left[\boldsymbol{\Delta}^{i}\right]$ and at least one $B \in\left[\mathbf{S}^{i}\right]$. Considering each coordinate, this interpretation gives:

$$
\forall s \in\left[\mathbf{s}_{k}^{i+1}\right],\left(\forall b \in\left[\mathbf{s}_{k}^{i}\right], \exists \delta \in\left[\delta_{k}^{i}\right]\right) \text { or }\left(\forall \delta \in\left[\delta_{k}^{i}\right], \exists b \in\left[\mathbf{s}_{k}^{i}\right]\right) \text { such that } \delta=s-b
$$

with $\left[\mathbf{a}_{k}\right]$ being the $k^{\text {th }}$ coordinate of a vector $[\mathbf{A}] \in I V$, that is $\left[\mathbf{s}_{k}^{i+1}\right]$ is the solution either of $\left[\delta_{k}^{i}\right]=\left[\mathbf{s}_{k}^{i+1}\right] \oplus\left[-\mathbf{s}_{k}^{i}\right]$ or of $\left[\mathbf{s}_{k}^{i}\right]=\left[\mathbf{s}_{k}^{i+1}\right] \oplus\left[-\delta_{k}^{i}\right]$. As shown in Section 2.3, the solution of this set of equations is given by $\left[\mathbf{S}^{i+1}\right]=\left[\mathbf{S}^{i}\right] \boxplus\left[\boldsymbol{\Delta}^{i}\right]$.

Practically speaking, the convergence of this method highly depends on the value of the real factor $\lambda$. Contrary to the precise-valued case, the convergence does not seem to be achieved for any value of $\lambda$ in $(0,1]$.

In iterative inversion procedures, the question often arises as to when to stop the iterative process (i.e. how to detect the convergence)? For precise-valued estimations, one possible criterion is the distance between $M$ and $A S^{i}$. When the equation has an exact solution, then $\left\|M-A S^{i}\right\|$ can be compared to the computation precision in order to stop the process. However, when the process is noisy because the measurements are noisy or because the model is imprecise then after a convergence period, the estimated value diverges from the "true" value.

In the interval-valued case we propose, there is another answer to this question. In fact, if the noise comes only from the imprecision of the model (i.e. the phenomenon we model) then a kind of adequacy convergence can be easily detected by the fact that, for certain iterations $i, M \in\left[\underline{M}^{i}, \bar{M}^{i}\right]$.

Since we have $\left[\underline{M}^{i}, \bar{M}^{i}\right]=\sigma_{h} \cdot \mathcal{A}_{\nu}\left(\left[\mathbf{S}^{i}\right]\right)$, according our construction, $\left[\underline{M}^{i}, \bar{M}^{i}\right]$ is the union of all the values $\sigma_{h} A_{P} S$ with $S \in\left[\mathbf{S}^{i}\right]$ and $P \in \operatorname{core}(\nu)$. So a simple interpretation of the adequacy convergence criterion is: there is at least one $P$ in $\operatorname{core}(\nu)$ and one $S$ in $\left[\mathbf{S}^{i}\right]$ such that $M=\sigma_{h} A_{P} S$. Stopping the reconstruction when the adequacy convergence is reached means that there is a solution in the obtained interval-valued reconstructed signal that corresponds to an impulse response in the considered set of impulse responses.

\subsection{Properties}

Definition 6 The interval-valued inversion process is said to be adequacy convergent if there is an index $p$ such that $M \in\left[\underline{M}^{p}, \bar{M}^{p}\right]$.

Proposition 1 The adequacy convergence defined in Definition 6 is equivalent to $\exists p \in \mathbb{N}$ such that $0 \in\left[\boldsymbol{\Delta}^{p}\right]$, with 0 being the vector equal to 0 everywhere.

\section{Proof:}


- If, for an index $p, M \in\left[\mathbf{M}^{p}\right]$, thus $\exists S \in\left[\mathbf{S}^{p}\right]$ such that $\exists P \in \operatorname{core}(\nu)$ such that $M-\sigma_{h} \mathcal{A}_{P}(S)=0$. Note now that $\mathcal{A}_{\nu^{T}}(0)=0$ implies $0 \in\left[\boldsymbol{\Delta}^{p}\right]$.

- If, for an index $p, 0 \in\left[\boldsymbol{\Delta}^{p}\right]$ then, for each coordinate $k, \exists P \in \operatorname{core}(\nu)$ and $\exists S \in\left[\mathbf{S}^{p}\right]$ such that $B=\sigma_{h} A_{P} S$ and $m_{k}-b_{k}=0$ and thus $M \in\left[\mathbf{M}^{p}\right]$.

Definition 7 The interval-valued inversion process is said to be completely adequacy convergent if there is an index $p$ such that $\forall i \geq p, M \in\left[\underline{M}^{i}, \bar{M}^{i}\right]$.

Proposition 2 The complete adequacy convergence is equivalent to $\exists p \in \mathbb{N}$ such that $\forall i \geq p 0 \in\left[\boldsymbol{\Delta}^{i}\right]$, with 0 being the vector equal to 0 everywhere.

Proof: For this property, the equivalence proved in Proposition 1 is statisfied for all indexes $i \geq p$.

The non-linear inversion process we propose leads to a sequence of intervals. Convergence of this sequence to its interval-valued limit is the solution of Equation 17, and corresponds to what we call the complete convergence of the process.

Definition 8 The interval-valued inversion process is said to be completely convergent if there is an index $p$ such that $\forall i \geq p,\left[\mathbf{S}^{i+1}\right]=\left[\mathbf{S}^{i}\right]$.

Note that if $p$ exists such that $\left[\mathbf{S}^{p+1}\right]=\left[\mathbf{S}^{p}\right]$ then for all $i \geq p$ we have $\left[\mathbf{S}^{i+1}\right]=\left[\mathbf{S}^{i}\right]$. Complete convergence of the iterative inversion procedure implies the adequacy convergence and more precisely we have the following result.

Proposition 3 The following assertions are equivalent:

- $\exists$ s such that $\left[S^{p+1}\right]=\left[S^{p}\right]$

- $\left[\Delta_{k}^{p}\right]$ the $k$-th coordinate of $\left[\Delta^{p}\right]$ is either 0 or the interval centred on 0 with a radius twice the radius of $\left[S_{k}^{p}\right]$.

Proof: If $\exists p$ such that $\left[S^{p+1}\right]=\left[S^{p}\right]$ then $\left[S^{p}\right]$ is solution of the equation $[\mathbf{X}]=[\mathbf{X}] \boxplus[\boldsymbol{\Delta}]$.

For each coordinate $k$, the previous condition entails either $\left[\delta_{k}\right]=\left[\mathbf{x}_{k}\right] \oplus\left[-\mathbf{x}_{k}\right]$ or $\left[\mathbf{x}_{k}\right]=\left[\mathbf{x}_{k}\right] \oplus\left[\delta_{k}\right]$.

- If $\left[\delta_{k}\right]=\left[\mathbf{x}_{k}\right] \oplus\left[-\mathbf{x}_{k}\right]=\left[\underline{x}_{k}-\bar{x}_{k}, \bar{x}_{k}-\underline{x}_{k}\right]$, then $\left[\delta_{k}\right]$ is an interval centred on 0 . Moreover, the radius ${ }^{3}$ of interval $\left[\delta_{k}\right]$ is twice the radius of interval $\left[\mathbf{x}_{k}\right]$.

- If $\left[\mathbf{x}_{k}\right]=\left[\mathbf{x}_{k}\right] \oplus\left[\delta_{k}\right]=\left[\underline{x}_{k}-\bar{\delta}_{k}, \bar{x}_{k}-\underline{\delta}_{k}\right]$, then $\bar{\delta}_{k}=\underline{\delta}_{k}=0$.

Reciprocally, if $\left[\Delta_{k}^{p}\right]$ is either 0 or the interval centred on 0 with a radius twice the radius of $\left[S_{k}^{p}\right]$ then, using the equation $\left[\mathbf{S}^{i+1}\right]=\left[\mathbf{S}^{i}\right] \boxplus\left[\boldsymbol{\Delta}^{i}\right]$, the first assertion is satisfied.

Note that complete convergence entails adequacy complete convergence.

$\left.\overline{{ }^{3} \text { with }[\mathbf{x}]=[x}, \bar{x}\right]$ being a real interval, its radius is defined by $\operatorname{rad}([\mathbf{x}])=\frac{1}{2}(\bar{x}-\underline{x})$ 
This implication scheme cannot be reversed. In fact, if for a particular iteration $p, 0 \in\left[\Delta^{p}\right]$ (i.e. adequacy convergence) due to the dual Minkowski operator, the fact that $0 \in\left[\Delta^{p+1}\right]$ cannot be guaranteed. In the same way, complete adequacy convergence does not lead to complete convergence, i.e. the fact that there is an iteration $p$ such that $\forall i \geq p 0 \in\left[\Delta^{i}\right]$ does not imply that the imprecision of the interval valued reconstructed signal is constant. The conditions that both signal and capacity have to fulfill to achieve one of these convergences of the algorithm (adequacy, complete adequacy, complete) require further study.

\section{Experimentation}

The two experiments we propose aim at illustrating the different properties we mentioned and highlight a certain number of other properties. This experiment is based on simulating a signal acquisition via a sensor whose discrete impulse response has been identified but with no information about the sampling kernel ensuring the continuous to discrete interplay. The discrete impulse response of the sensor is depicted in Figure 3. The signal whose measurement is simulated is made of a weighted sum of 10 sine waves whose frequencies were randomly chosen. The signal we process is of high dimension (100000 samples) to comply with the hypothesis that the pseudo-inverse cannot be easily computed. The figures we present here only plot $10 \%$ of the signals.

We model the fact that the sampling kernel is unknown by using a capacity constructed with the procedure defined in [18]. In addition, we compute 40 different discrete impulse responses while hypothesizing 40 different sampling kernels. We reconstruct an imprecise valued estimate of the signal by using our interval valued iterative procedure and 40 precise valued estimates of the signal by using the conventional precise valued least squares iterative procedure. 50 iterations of the reconstructing processes were performed.

\subsection{Experimentation with no random noise}

Within the first experiment, the measurement process is supposed to be free of random variations, i.e. the measurement noise is only due to the fact that the sampling kernel is unknown. Figure 1 shows the superposition of the intervalvalued reconstructed signal (blue-upper, red-lower) with the true signal (dotted black) and the 40 precise least squares reconstructions using the 40 different discrete impulse responses (cyan). In Figure 1.a, all the reconstructed signals seem identical. 1.b plots a zoomed detail of this superposition. As can be seen in this last figure, all precise valued reconstructed signals are included in the imprecise valued reconstructed signal. This property is true for any iteration of the reconstruction process. It is the main motivation for this work, i.e. to be able to derive an interval-valued reconstruction process that includes all precisevalued reconstruction processes it models.

This inclusion has not yet been mathematically proven. If this property holds whatever the signal, it can be considered as a kind of robustness of the inversion 
process since, if the lack of knowledge on the impulse response of the sensor is properly modeled by the capacity (i.e. $\exists P \in \operatorname{core}(\nu)$ such that $M=\sigma_{h} A_{P} S$ ), then the inclusion of the signal in its interval-valued estimate seems to be guaranteed. Moreover, within this random-noise free experiment, the true signal is included in the interval-valued estimate of the signal while none of the 40 precise estimates of the signal have converged to the true signal. Inclusion of the true signal in its imprecise estimate is measured by computing the proportion of signal samples that are included in its interval valued reconstruction during the reconstruction process. The value of this proportion for each iteration is plotted in Figure 4.

The criterion to be minimized is the distance between the measurement vector $M$ and the modeled measurement of the interval valued reconstructed signal. As can be seen in Figure (2), this imprecise measurement includes the measurement vector and all precise measurements of the 40 precise valued reconstructed signals.

We also compute the proportion of measurement samples that are included in the imprecise measurement for each iteration. The value of this proportion versus the iterations is plotted in Figure 5. Within this experiment, what we call complete adequacy convergence is obtained after less than 10 iterations. The number of iterations that are necessary to obtain this convergence is not a constant of the method and varies with the experiments. Note however, that in a noise-free context, this convergence also corresponds to the total inclusion of the true signal in the interval-valued reconstructed signal (see Figure 4).

Finally, Figure 6 plots variations in the interval valued estimate mean imprecision (i.e. the mean of the interval valued estimate length) versus the iterations. It can be easily seen that this mean imprecision converges (which is true when the number of iterations tends to infinity).

\subsection{Experimentation with random additive noise}

The second experiment is performed by adding centered Gaussian random noise with a standard deviation of 0.8 to the measurement samples, i.e. the signal-tonoise ratio is about $50 \mathrm{~dB}$. When the measurement is noisy, then the reconstruction cannot be exact. In this case, the Schultz procedure converges towards the least squares solution. Moreover, as usual, the reconstructed signal diverges from the true signal, since this procedure is not regularized. In this experiment, the divergence occurs after 20 iterations, as illustrated in Figure 9. The measurement samples are also not included in their interval-valued estimates, as illustrated in Figures 8 and 10 .

When variations in the measurements are not accounted for by the capacitybased imprecise representation of the discrete impulse response, which is the case here, the desired inclusion property is no longer ensured. In fact, after a certain number of iterations, the least squares solutions obtained when considering impulse responses that are dominated by the considered capacity are no longer completely included in the interval-valued reconstruction. As a matter of fact, 


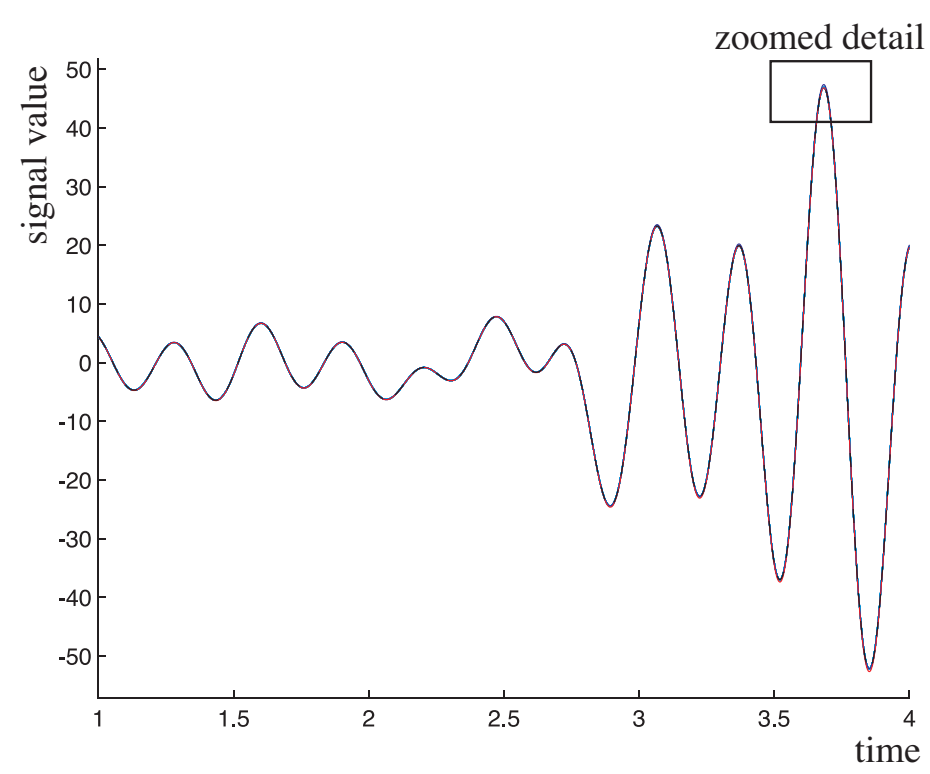

a)

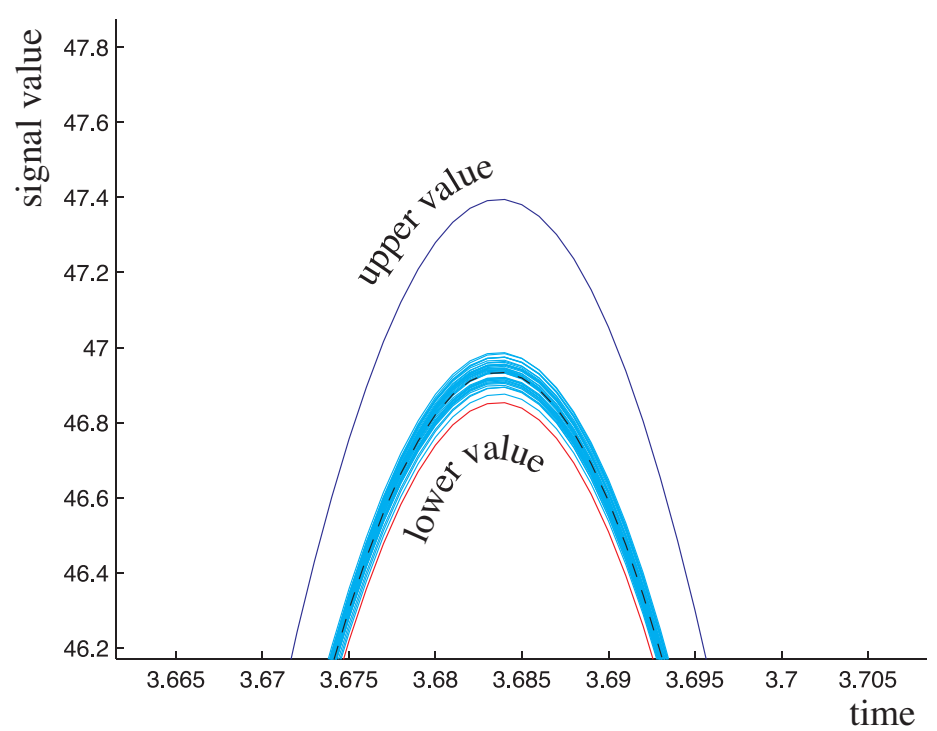

b)

Fig. 1. Interval-valued reconstructed signal (blue upper, red lower) superimposed with 40 precise-valued reconstructed signals (cyan) and the original signal (dotted black), without additive random noise (b: detail of the superposition) 


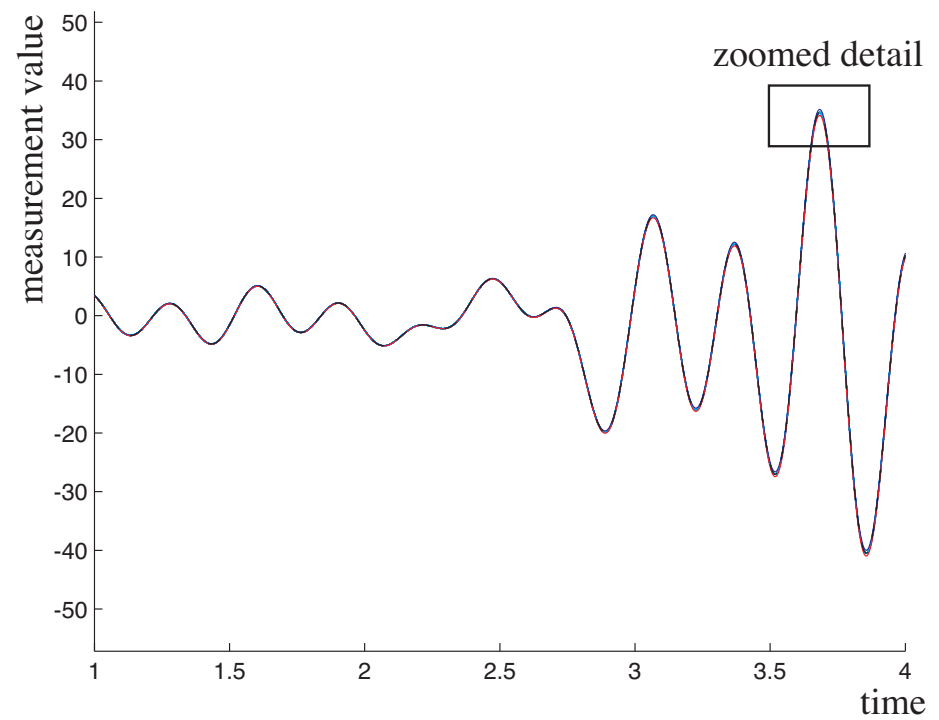

a)

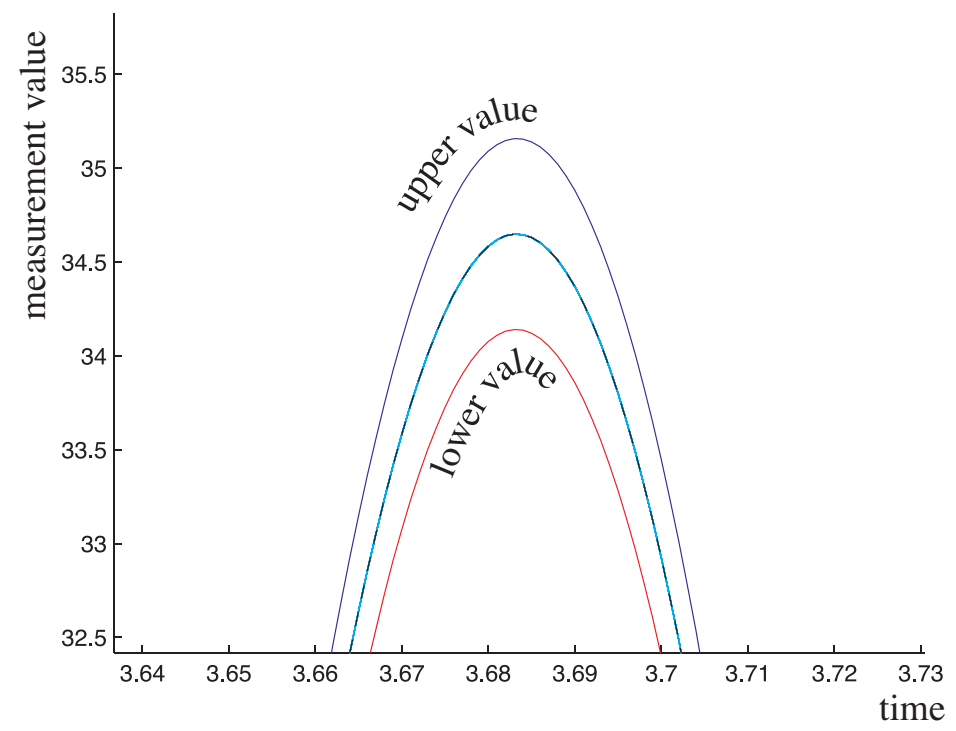

b)

Fig. 2. Interval-valued reconstructed measurement (blue upper, red lower) superimposed with 40 precise-valued reconstructed measurements (cyan) and the original measurement (dotted black), without additive random noise (b: detail of the superposition) 


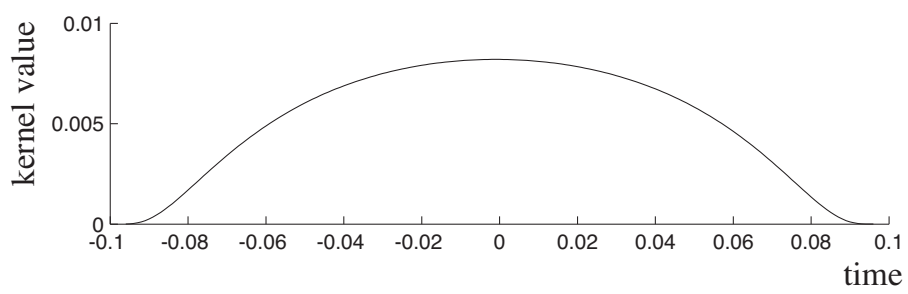

Fig. 3. The impulse response of the sensor

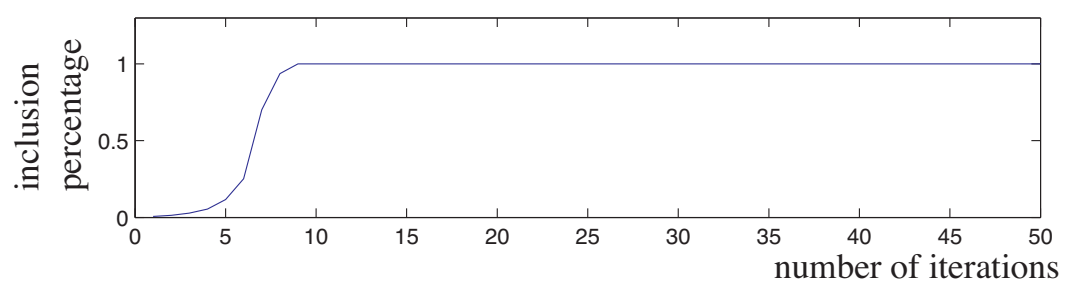

Fig. 4. Ratio of signal samples included in the interval valued signal estimate

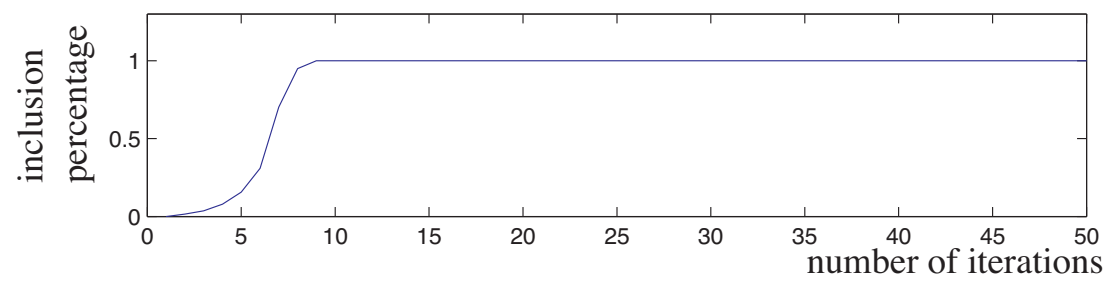

Fig. 5. Ratio of measure samples included in the interval valued measure estimate

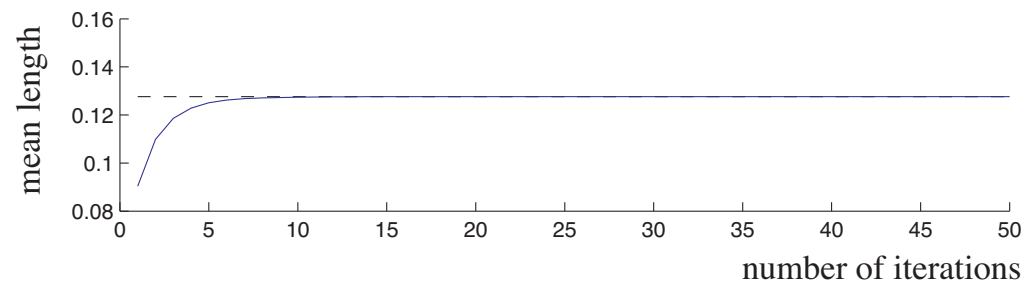

Fig. 6. Variations in the reconstructed interval length 
the inclusion property seems to be linked with least squares convergence and divergence, as illustrated in Figures 7, 9 and 13. Figure 7 plots the interval-valued reconstructed signals superimposed with 40 precise-valued reconstructed signal after 15 iterations of the reconstruction process. For 15 iterations, the inclusion property is verified (but the true signal is not included in the interval-valued reconstructed signal). Figure 13 plots same superposition after 2000 iterations of the reconstruction process. In that case, every least squares solution has completely diverged and is far from the true signal. This divergence is illustrated in Figure 9: after 18 iterations, the number of signal samples included in the interval-valued reconstruction decreases when the number of iterations increases. On the other hand, the 40 precise estimates of the measures are always included in the imprecise estimate of the measures whatever the number of iterations (see Figures 8 and 12).

A way to regularize an iterative reconstruction process is to stop a nonregularized iterative reconstruction process when a convergence criterion is verified. However, finding a robust criterion to achieve this interruption is still an open problem [22]. Within our interval-based reconstruction process, a very simple regularization can be achieved by stopping the reconstruction process as soon as the inclusion of the measurement in the interval valued estimate of the measurement is stabilized (which is obtained here after the $18^{\text {th }}$ iteration - see Figure 10). Figure 11 seems to show that the mean length of the interval-valued estimate is also stabilized after 18 iterations. This interpretation is wrong. In fact, the mean length of the interval-valued estimate slowly increases with the iterations, highlighting the fact that the modeling is not suitable for this problem (since the least squares process is not regularized).

Moreover, the fact that the adequacy convergence is never verified (see Figure 10) can be considered as a criterion for detecting poor conditioning of the considered inverse problem. When continuing the reconstruction process, the interval-valued estimate of the measurement tends to include the measurement while its imprecision slowly increases, as illustrated in Figure 12. At the same time, the interval-valued estimate of the signal diverges from the true signal by adding artifacts (see Figure 13). Knowledge on the measurement noise can perhaps be included by considering the measured samples as being interval valued (with the interval valuation accounting for a known confidence interval).

As a last remark, it can be experimentally highlighted that the noise-quantification ability of this kind of method, as mentioned in [15], seems to apply. That is, the imprecision of the interval-valued estimate is a marker of the impact of the measurement noise on the estimation. This last interesting property should be experimentally and mathematically studied in a future work.

\section{Conclusion and discussion}

When reconstructing a signal from its measurements by using a deconvolution process, the fact that the impulse response of the acquisition system is ill-known is barely taken into account. Most papers focus on the measurement random 

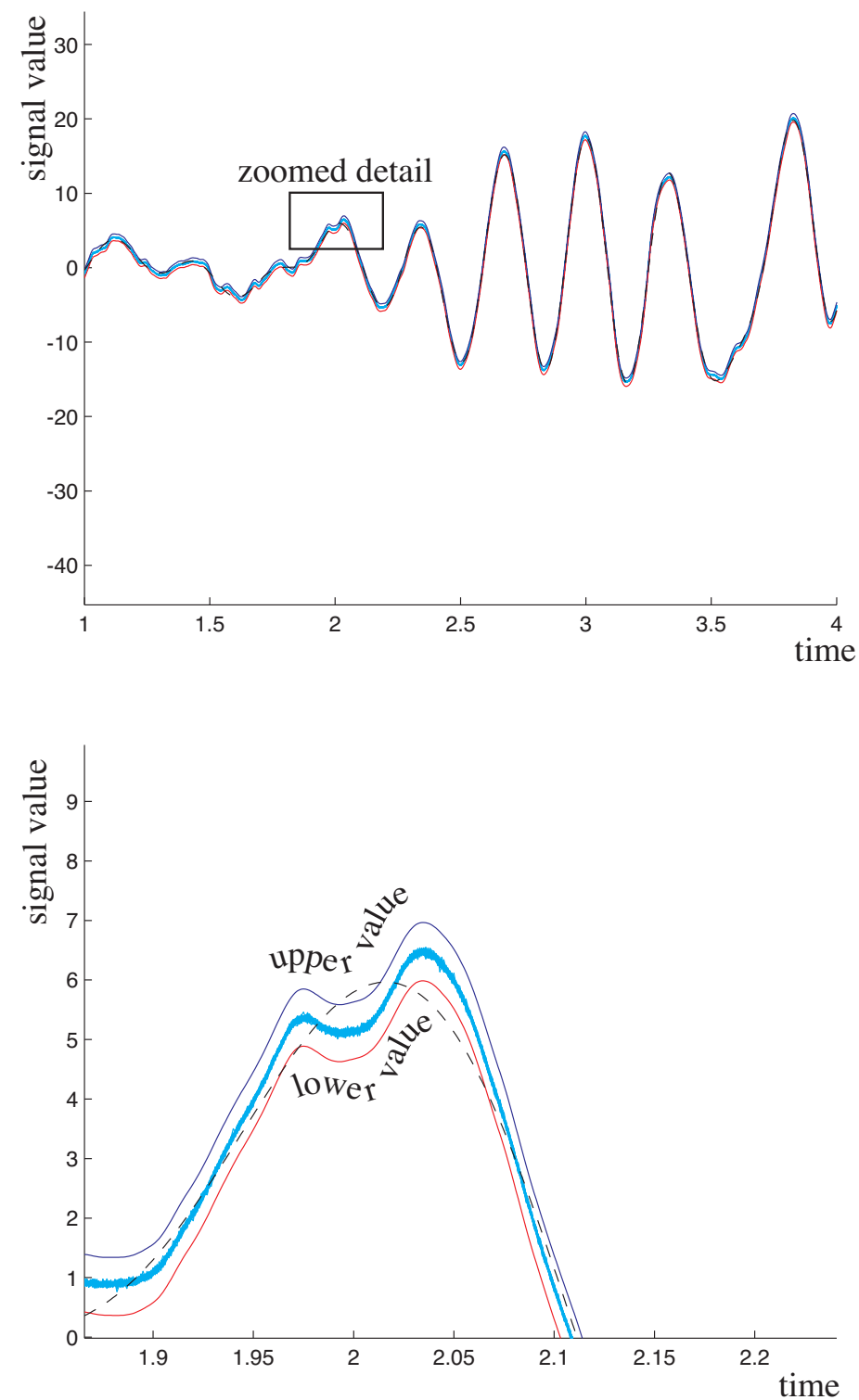

Fig. 7. Interval-valued reconstructed signal (blue upper, red lower) superimposed with 40 precise-valued reconstructed signals (cyan) and the original signal (dotted black), with additive random noise (b: detail of the superposition) 

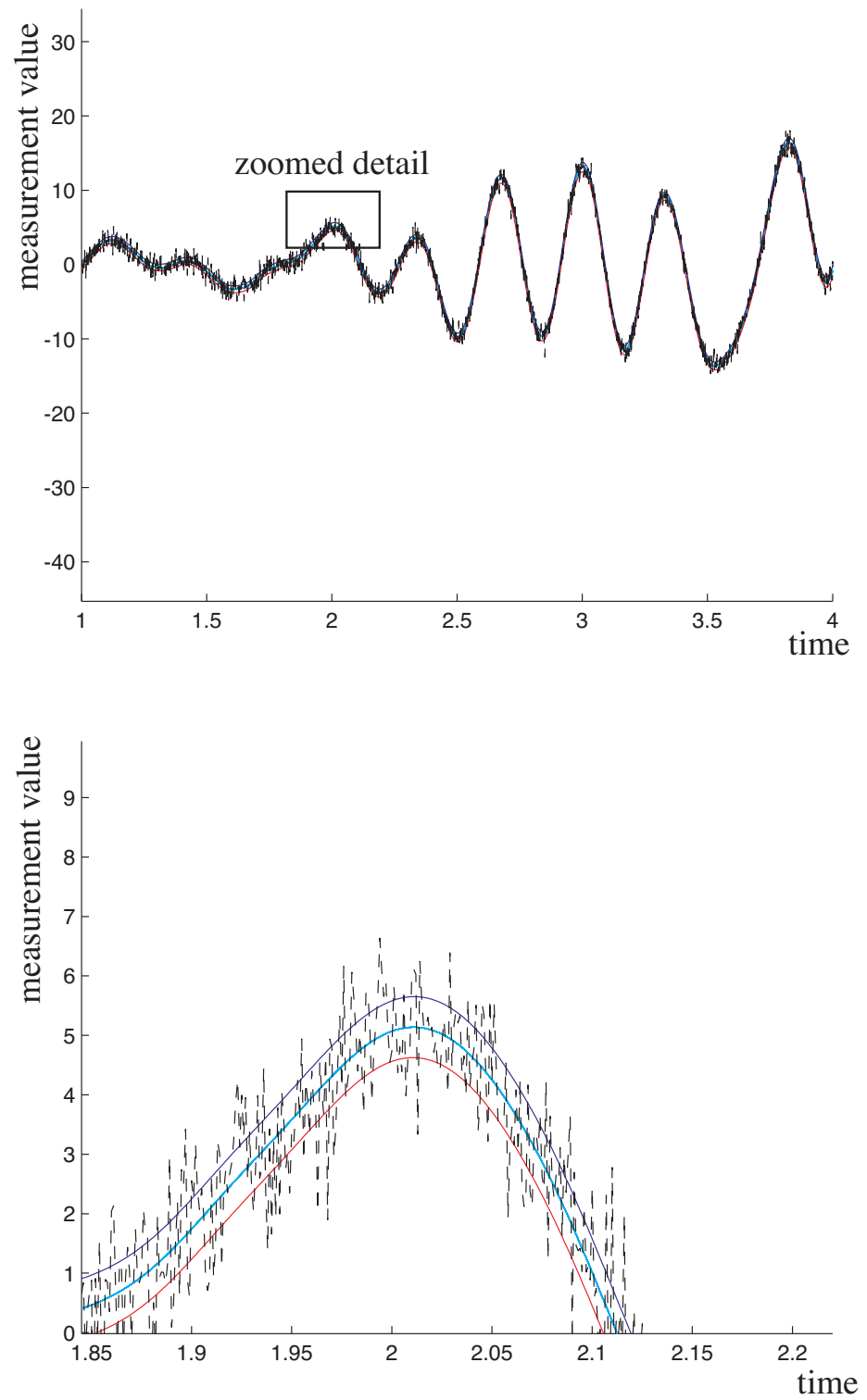

Fig. 8. Interval-valued reconstructed measurement (blue upper, red lower) superimposed with 40 precise-valued reconstructed measurements (cyan) and the original measurement (dotted black), with additive random noise (b: detail of the superposition) 


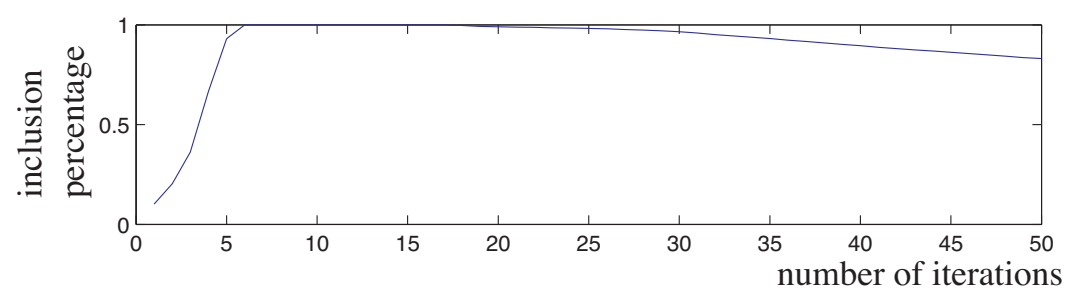

Fig. 9. Ratio of signal samples included in the interval valued signal estimate

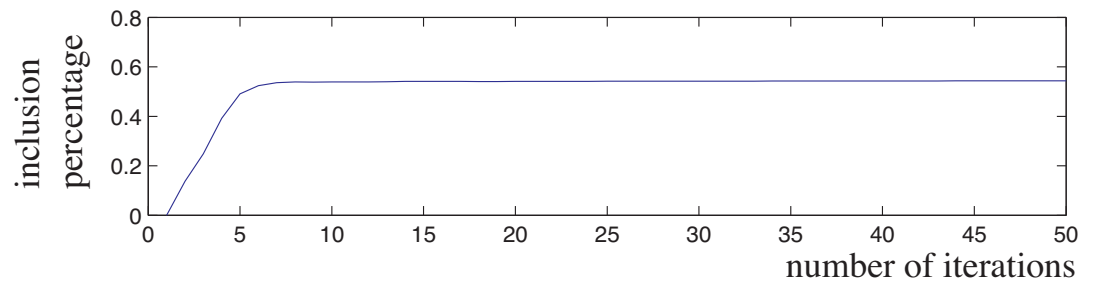

Fig. 10. Ratio of measure samples included in the interval valued measure estimate

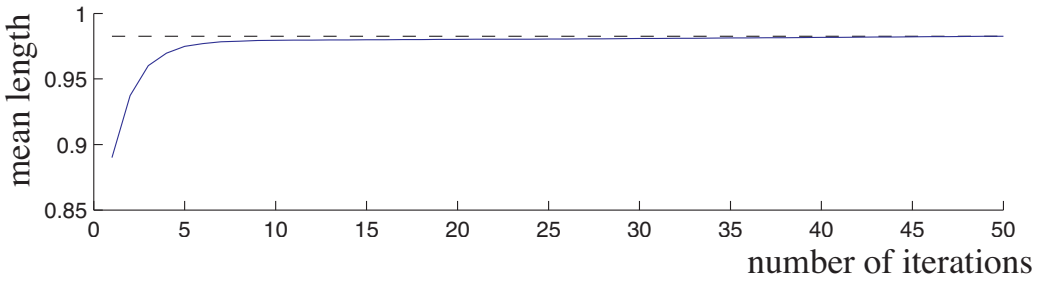

Fig. 11. Variations in the reconstructed interval length 


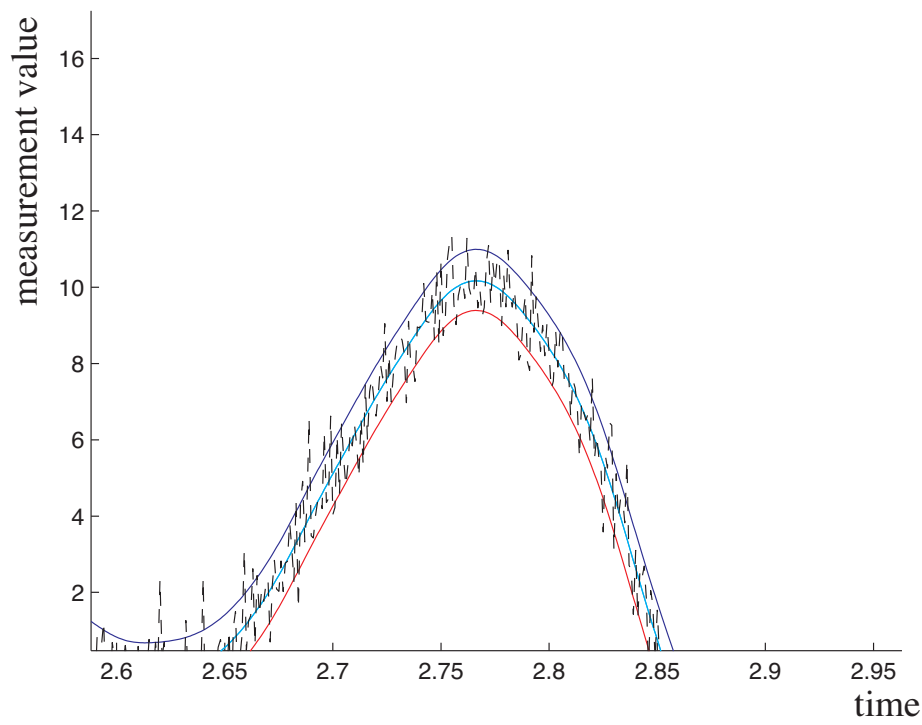

Fig. 12. Detail of the measure estimated interval after 2000 iterations

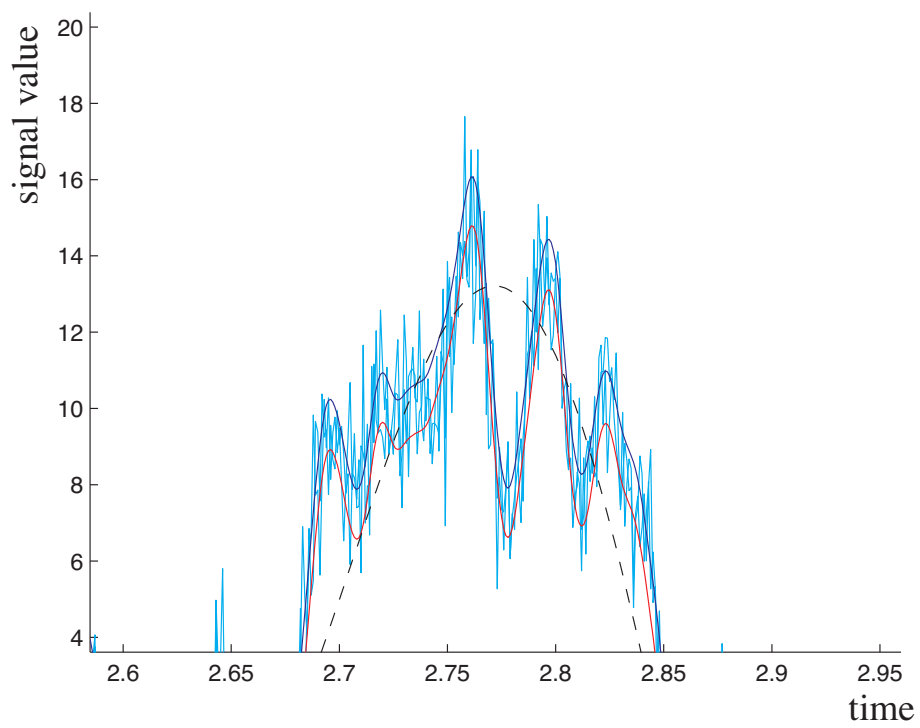

Fig. 13. Detail of the signal estimated interval after 2000 iterations 
noise. In [18] we have proposed a very simple modeling of this ill knowledge by considering a whole convex set of imprecise responses and replacing the precise convolution operation by an imprecise convolution operation. The obtained imprecise output is simply the convex hull of all outputs that should have been obtained by a precise convolution operator with different modeled impulse responses. In this paper, we have proposed an inversion of this model, which leads to an imprecise estimate of the original signal knowing its measurement and a set of possible impulse responses of the sensor. A certain number of properties of this procedure have been proved while others have just been highlighted via some experiments.

This pioneer work leads to more questions than answers. For example:

- is the reconstructed signal the most specific one that verifies the desired properties?

- can this approach be extented to impulse responses having positive and negative values?

- what is the relation between this kind of method and the usual guaranteed interval-based approach?

- is it possible to also consider that the gain in the impulse response is also imprecise?

- is it possible to consider any concave capacity?

- is it possible to add a regularization in this procedure and thus decrease artifacts due to random noise?

- is it possible to account for knowledge on the random measurement noise?

It should now be of prime importance to test this kind of deconvolution in applications, in order to see wether such an interval base approach leads to improvement in signal analyses or not.

Moreover, if it appears that solving this kind of problem is instrumental in signal processing applications, it would be relevant to envisage other generalizations. In fact, in the precise valued linear signal processing context, deconvolving by using the Schultz iterative procedure is neither the most effective nor the fastest solution. Other methods could be used like a singular value decomposition of matrix $A_{P}$ with zeroing the smallest eigenvalues, or an expectation-maximization strategy based on an appropriate cost function. Such approaches have been successfully used for inverting measurement equations involving interval-valued matrices. Our modeling of the measurement equation does not come within this framework, so extending the singular value decomposition approach would require reinterpretation of the concept of eigenvalues and/or eigenvectors. Extending the expectation-maximization approach requires an appropriate cost function and a generalization of the corresponding iterative algorithm.

Thus, the global problem remains open. What we have proposed here is just a convenient, effective and simple solution. 


\section{References}

1. L. Campos, J. Huete, and S. Moral. Probabilities intervals: a tool for uncertain reasoning. International Journal of Uncertainty, Fuzzyness and Knowledge-Based Systems, 2:167-196, 1994.

2. G Choquet. Theory of capacities. Ann. Inst. Fourier, 5:131-295, 1953.

3. D. Denneberg. Non-Additive Measure and Integral. Springer, 1994.

4. Didier Dubois. Possibility theory and statistical reasoning. Computational Statistics Ef Data Analysis, 51(1):47-69, November 2006.

5. P. Eggermont and G. Herman. Iterative algorithms for large partitioned linear systems with applications to image reconstruction. Linear algebra and its applications, 40:37-67, 1981.

6. M Gerver. The inverse problem of seismology. Tectonophysics, 13:483-496, 1972.

7. $\mathrm{P}$ Gilbert. Iterative methods for the three-dimensional reconstruction of an object from its projections. J. Theor. Bio., 36:105-117, 1972.

8. R. Gordon, R. Bender, and G. Herman. Algebraic reconstruction techniques (art) for three dimensional electron microscopy and x-ray photography. J. Theor. Bio., 36:105-117, 1970.

9. M. Grabisch and Ch. Labreuche. The symmetric and asymmetric Choquet integral on finite spaces for decision making. Satistical Papers, 43:37-52, 2002.

10. J. Herzberger and L Petkovi. Efficient iterative algorithms for bounding the inverse of a matrix. Computing, 44:237-244, 1990.

11. A Jalobeanu, L Blanc-Faud, and J Zerubia. Hyperparameter estimation for satellite image restoration using a mcmc maximum-likelihood method. Pattern Recognition, 35:341-352, 2002.

12. L. Jaulin, M. Kieffer, O. Didrit, and E. Walter. Applied interval analysis with exemples in parameter and state estimation. Robust Control and Robotics, 2001. Springer.

13. J. Kalifa and S. Mallat. Thresholding estimators for linear inverse problems and deconvolutions. Annals of Statistics, 1:58-109, 2003.

14. K. Loquin and O. Strauss. On the granularity of summative kernels. Fuzzy Sets and Systems, 159(15):1952-1972, 2008.

15. K. Loquin and O. Strauss. Noise quantization via possibilistic filtering. In International Symposium on Imprecise Probability: Theory and Applications, ISIPTA 2009, page 297-306, Durham, 2009.

16. M. Hudson and T. Lee. Maximum likelihood restoration and choice of smoothing parameter in deconvolution of image data subject to poisson noise. Computational Statistics and Data Analysis, 26(4):393 - 410, 1998.

17. J. Rice. Choice of smoothing parameter in deconvolution problems. Contemporary Mathematics, 59:137-151, 1986.

18. A. Rico and O. Strauss. Imprecise expectations for imprecise linear filtering, International Journal of Approximate Reasoning, 51: 933-947, 2010.

19. L. Shepp and Y. Vardi. Maximum likelihood reconstruction for emission tomography. IEEE Trans. Med. Imaging, 2:113-122, 1982.

20. D. Schmeidler, Subjective probability and Expected utility without additivity, Econometrica vol 57, 571-587.

21. M. Unser. Splines: A perfect fit for signal and image processing. IEEE Signal Process. Mag., 16:22-38, 1999.

22. D. Mariano-Goulart and P. Maréchal and S. Gratton and L. Giraud and M. Fourcade A priori selection of the regularization parameters in emission tomography by Fourier synthesis. Comput. Med. Imaging Graph., 31:502-9, 2007. 